
\section{BMJ Quality}

\title{
Speeding up laboratory test reporting in Medical Emergency and Cardiac Arrest calls: a quality improvement project
}

\author{
Mohammed Al-Talib, Isla Leslie
}

To cite: Al-Talib M, Leslie I. Speeding up laboratory test reporting in Medical Emergency and Cardiac Arrest calls: a quality improvement project. BMJ Quality Improvement Reports 2017;6:u213103.w5207. doi:10.1136/bmjquality. u213103.w5207

Received 11 August 2016 Revised 12 December 2016

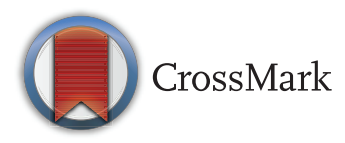

Brighton and Sussex University Hospitals Trust, UK

Correspondence to Mohammed Al-Talib maltalib27@gmail.com

\section{ABSTRACT}

Many hospitals deploy Medical Emergency (MET) and Cardiac Arrest teams to improve the management and treatment of patients who become critically ill. In many cases, blood results are key in allowing the clinicians involved in these teams to make definitive management decisions for these patients. Following anecdotal reports that these results were often delayed, we assessed the process of blood tests being reported in emergency calls, identified the key factors causing delays and sought to make improvements.

The initial intervention involved implementing a new blood form that specified the nature of the call, the tests required and a contact number for laboratory staff to contact the clinical team with results. We also developed a streamlined process within the laboratory for these samples to be fast-tracked. Successive improvement cycles sought to increase awareness of the project, improve accessibility to the new forms and embed spontaneous practices that contributed to improvement.

Results demonstrated an overall reduction in the time taken for blood samples in emergencies to be reported from 130 minutes to 97 minutes.

This project demonstrates that using a specific blood request form for emergency calls, and tying this to a specified laboratory process, improves the time taken for these tests to be reported. In addition, the project provides some insight into challenges faced when implementing change in new departments.

\section{PROBLEM}

'Medical Emergency' and 'Cardiac Arrest' teams are deployed in a number of hospitals in the UK. Usually comprising of a medical registrar, medical house officers, an intensivist or anaesthetist and critical care nurses; this team manages the treatment of patients who become acutely unwell on a hospital ward.

The Royal Sussex County Hospital (RSCH, Brighton, UK) has over 500 beds and serves as a tertiary referral centre in South-east England. There is no strict definition or criteria for a medical emergency at RSCH, with the emphasis upon staff that a call should be put out for any patient they are acutely concerned about. This typically correlates with a high NEWS (National Early Warning Score) score.

During the initial assessment and investigation of these patients, basic laboratory tests are invariably performed. The results of these tests are often vital in establishing a diagnosis and guiding a management plan. As such, a positive outcome for the patient may rely upon prompt reporting of these tests. Anecdotal reports from medical staff at RSCH suggested that laboratory test results for these critically ill patients were often slow to be reported.

As such two members of the medical team (Foundation Year 2 doctors) determined to identify the factors contributing to delays and design interventions to resolve them. Given the importance of haste, our initial SMART aim was to speed up the reporting of laboratory tests by for patients who are in a 'medical emergency' or 'cardiac arrest' by $50 \%$ over a 2 month period, and for this improvement to be sustained.

\section{BACKGROUND}

It is widely accepted that the use of Medical Emergency (MET) teams in hospitals has a positive impact on patient safety and outcomes. ${ }^{12}$ Although data on the actual effectiveness of this approach in reducing mortality is unclear, the deployment of these teams is regarded as "scientifically rational". 3 For example, rapid diagnosis and treatment of sepsis is associated with significantly better outcomes. ${ }^{4}$

Procedures to hasten the turnaround time of laboratory tests have been deployed for Emergency Departments, specifically to improve throughput ${ }^{5} 6$ and, in the UK, to facilitate decision-making within the requisite 4-hour window. For example, point-of care testing has been shown to alleviate the effects of overcrowding on patient safety. ${ }^{7}$ 
At the Royal Sussex County Hospital, samples received from the Emergency Department are fast-tracked to achieve a turnaround time of less than one hour.

To determine whether laboratory tests in emergencies can be hastened, an understanding is needed of the factors, both technical and non-technical, that contribute to delay.

\section{MEASUREMENT}

The approach to baseline measurement in this project centred on two key areas. Firstly, outcome measurement of the actual time taken for laboratory tests for to be reported in MET and Cardiac Arrest calls. Secondly, structural measurement of each step in the process involved in laboratory tests being reported. Calculation of these two baseline measurements could then facilitate identification of key areas where quality improvement interventions can be focused.

To assess the time taken for laboratory test reporting in MET and Cardiac Arrest calls, a database of all emergency calls was used. This database was regularly updated by members of the hospital resuscitation team and included the date, time and nature of the call. A total of 166 emergency calls were identified between 15th December 2015 and 15th March 2016. Laboratory results for these patients were then identified using the hospital electronic reporting system. This system includes the times that samples are taken, received in the lab and reported on the system. By comparing these times against the time of the emergency call, we could assess an overall time taken for tests to be reported and, also, the time taken at each individual step of the process. The results showed a median reporting time of 130 minutes (see Graph 1).

The second aspect of baseline measurement was to develop an understanding of the processes involved in laboratory test reporting. This was done by using process mapping, a diagrammatic scheme of representing all the parallel processes in a given system. To create this process map, an observer undertook informal discussions with clinical, support and laboratory staff to gain a better understanding of the processes involved. The same observer then attended emergency calls to follow laboratory samples from the patient to reporting in the lab. As well as revealing the general process, this also allowed any variability in the process to be revealed.

\section{DESIGN}

The data from the baseline measurement, in addition to discussion with stakeholders, revealed a number of areas that could be amenable to improvement. Laboratory staff raised the issue of the current request forms including tests - such as for assessing thyroid function - which take considerably longer than other routine tests and delay the reporting of all results. Also, a contact number with which to relay results to a member of the clinical team was often not provided, delaying action being taken on grossly abnormal results. Taking into account the baseline measurements and discussions, a multipronged intervention was collaboratively designed to attempt to address the range of problem areas and bottlenecks identified.

We decided to limit our intervention to the RSCH rather than implementing it across other hospitals within the trust - the Royal Alexandra Children's Hospital (Brighton, UK) and the Princess Royal Hospital (Haywards Heath, UK). This was chiefly because we hoped to establish proof-of-principle that the project could work before expanding it to sites where we would be less able to directly identify problems.

We decided that at least one project member would attend emergency team morning meetings and emergency calls in the first two weeks. This would allow robust data collection with regards to timings of each step, and also for immediate troubleshooting of issues and redesign of interventions in subsequent PDSA cycles.

We planned to measure the effect of our intervention and subsequent PDSA cycles by collecting data on the times taken for blood tests to be reported for all

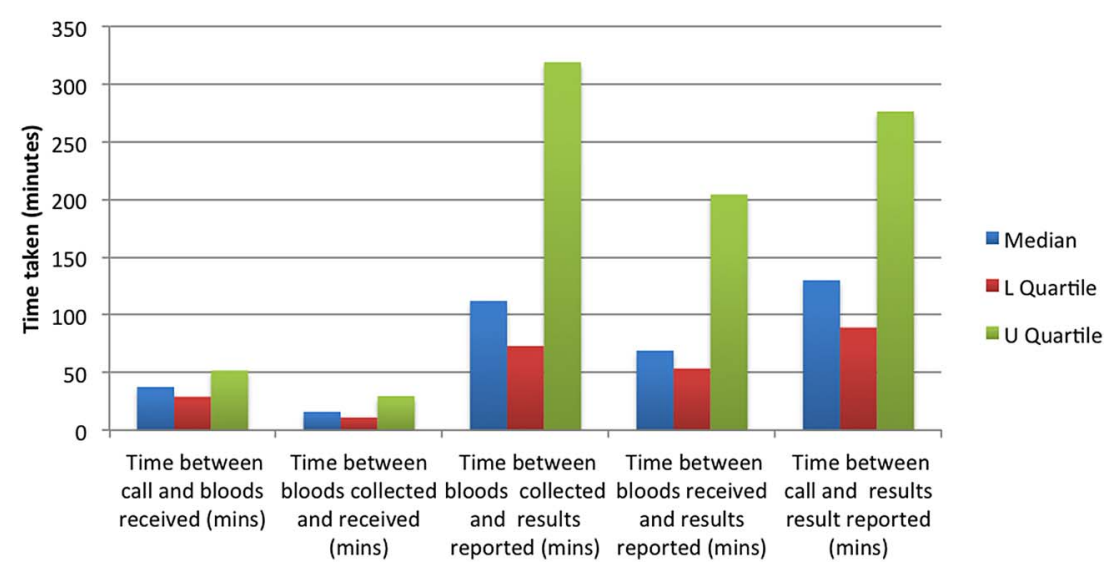


emergency calls over a 2 month period. This information was updated weekly by the hospital Resuscitation team on a shared database that recorded the date and time of the call and the patient hospital identification number. Project members would review the database at two-weekly intervals and, by linking the patient data to the laboratory reporting system, could identify and record the times taken.

\section{STRATEGY}

PDSA cycle 1:

Our initial intervention involved implementation of a pre-printed emergency sticker on blood forms that would be carried by members of the Critical Care Outreach team and Resuscitation team. This sticker was easily identifiable and included a pre-printed battery of blood tests which could be rapidly performed. It also had a space for a pager number to be written so that emergency results could be relayed back to staff once available.

We also implemented two interventions at the laboratory to hasten results. First, the on-call biomedical scientist bleeps were added to the emergency pager system so that they would have warning of impending samples. Second, a system was put in place for any samples labelled with the emergency sticker to be fast-tracked for rapid testing. The streamlining effect of these interventions are illustrated in the process maps (see images 1 and 2).

After 2 weeks, these interventions had led to a $40 \%$ improvement in the median turnaround time for laboratory results to be reported. The vast majority of this improvement came from speeding up the time for samples to be analysed and reported.

However, we received feedback that the forms were not being brought to emergency calls at night as the designated staff members carrying the forms were not rostered. Laboratory staff were also concerned that they were occasionally staying on high alert following an emergency call, only for no bloods to be received, for example in a false alarm or if no bloods were taken. In addition, they regularly noted that no emergency contact number was provided on the forms.

PDSA cycle 2:

The aim of the second cycle was to iron out issues over availability of the form and complaints from the laboratory and assess sustainability of the project over the next 2 weeks. We gained support from the nighttime Nurse Practitioners to bring the emergency forms to calls at night, and updated the forms such that the pager number for the on-call medical SHO was pre-printed. We also implemented a 'stand down' time of one hour at the laboratory, after which staff could assume that no samples had been taken. We stopped regularly attended morning meetings and emergency calls in order to start gauging the sustainability of the project.

However, over the 2 week period following the implementation of these interventions we noticed a drop off in usage of the forms with only two being used despite a total of 10 emergency calls being put out. This was associated with an increase in reporting times to above

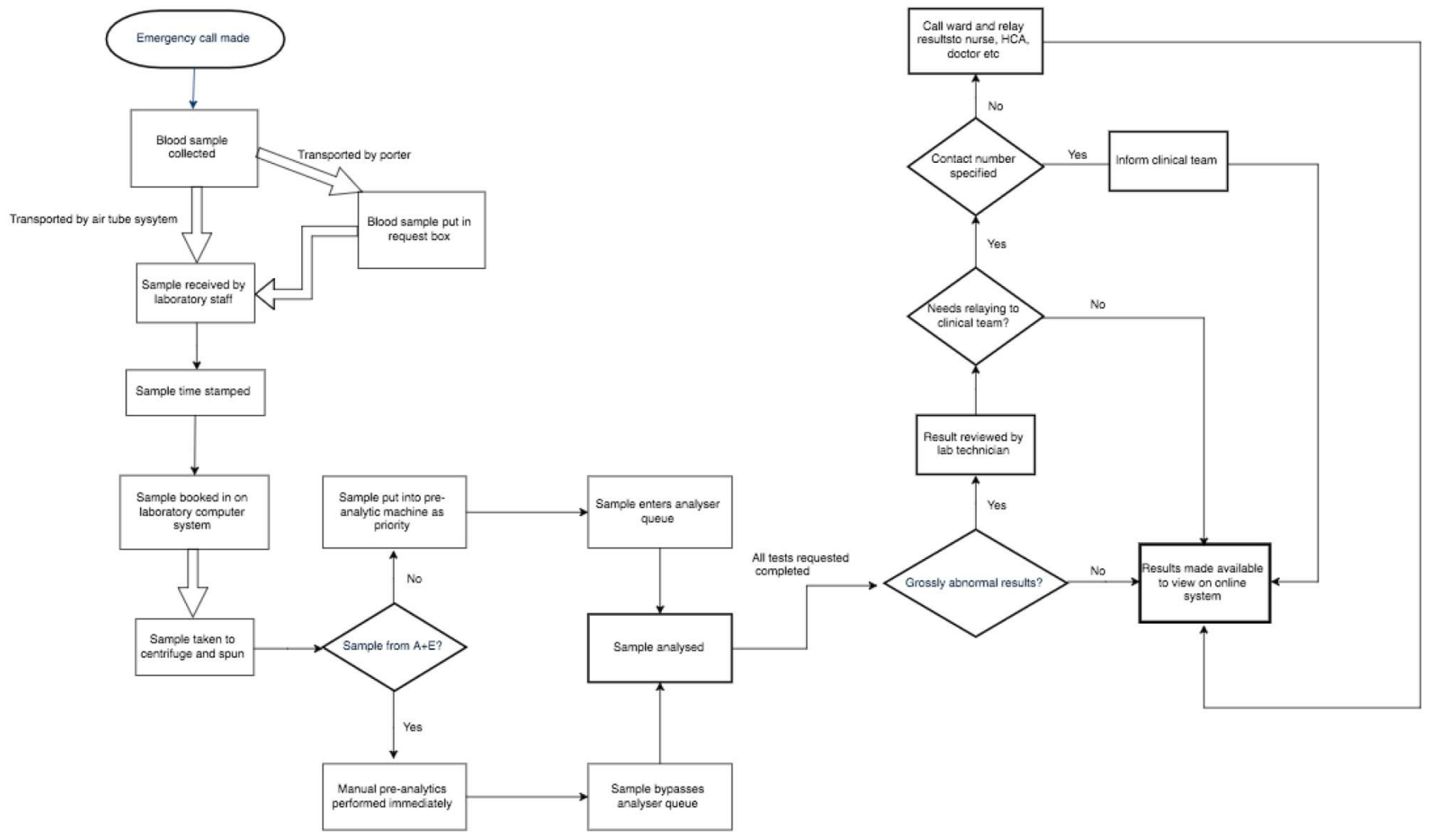

Image 1 process map of laboratory testing pre-intervention 


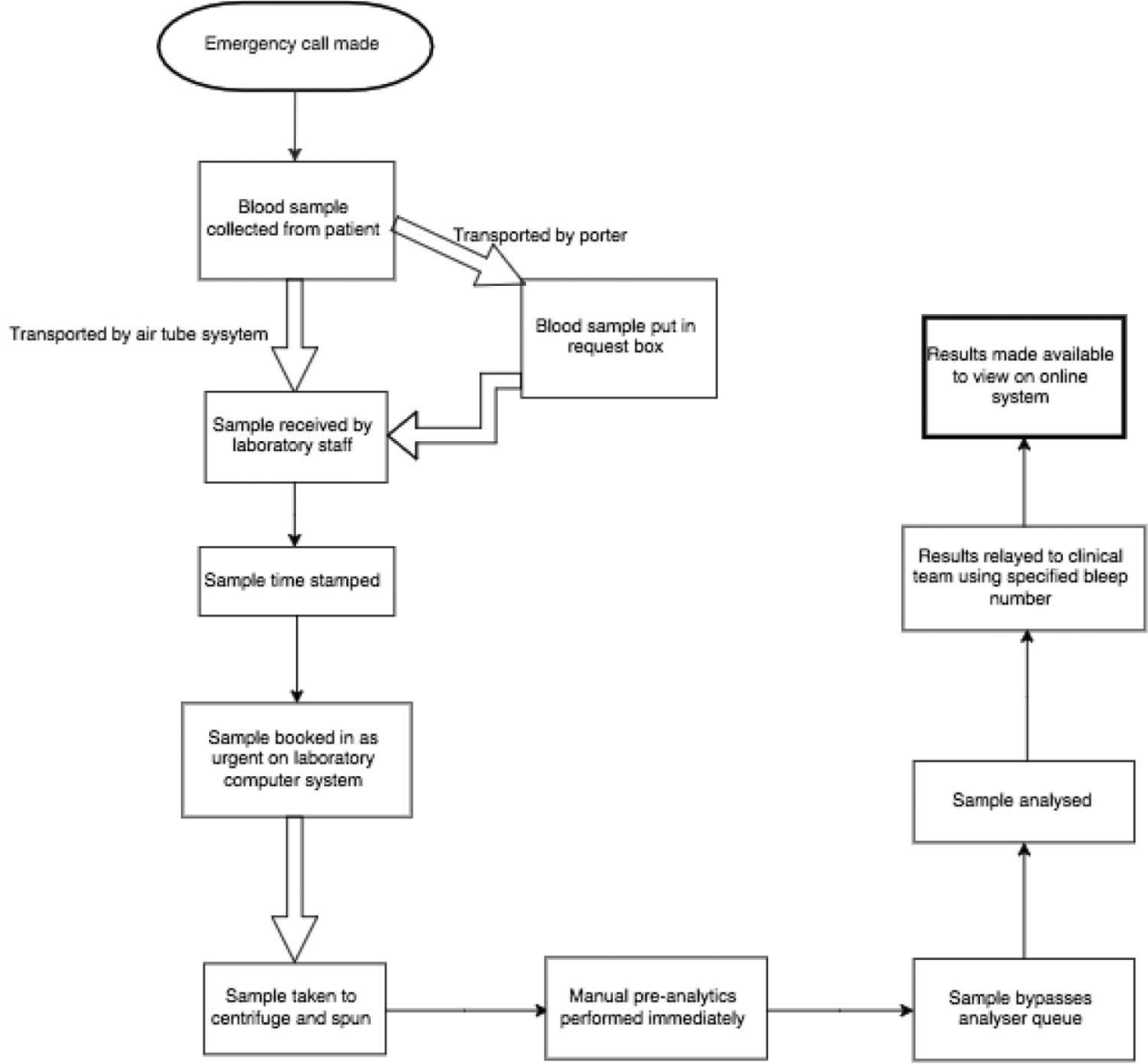

Image 2 process map of laboratory testing post-intervention

baseline levels. On discussion with clinical staff, the main reason appeared to be that they often forgot to use the forms in the high pressure situation, particularly as project members were no longer attending the morning meetings and emergency calls to remind them.

\section{PDSA 3:}

In order to embed the new process and practices, we sent regular email reminders to clinical staff and designed posters that highlighted the aims of the project and reminded staff exactly where forms could be found. We also modified the prompt card used in the emergency team morning meeting to add a specific point about reminding staff to use the forms. Coupled to this, we enlisted members of the Critical Care Outreach team to become champions for the project such that there would be further reminding for clinical staff.

We studied the impact of this cycle of interventions after 4 weeks and noted an increase in the usage of the forms that was coupled with an improvement in the reporting times to an average of 97 minutes, a $25 \%$ improvement from baseline.

\section{RESULTS}

The results of the project showed an overall reduction in reporting times for laboratory tests in emergency calls from a median of 130 minutes to 97 minutes. The greatest improvement was in the actual time taken for the laboratory to produce the results once they had received the samples (see graphs 2 and 3) - reflecting the new laboratory process we implemented. As discussed briefly above, this improvement extended to samples that had not used the new form but had specified that the bloods were taken in an emergency. There was no appreciable difference in the time taken for samples to be delivered to the lab following collection. Specifically considering cases where the form was used, the median reporting time was 81 minutes.

Following the considerable improvements in the first two weeks of implementation, there was a lengthening of reporting times for blood results, with a median time of 152 minutes in week 4 . This was clearly coupled with a drop in usage of the forms, with only 2 forms being used out of a total of 17 emergency calls over the preceding two weeks. Implementation of the reminder emails and posters, plus the update to the morning meeting prompt card, improved usage of the forms and, consequently, reporting times for blood results (117 minutes, 116 minutes, 76 minutes and 97 minutes in weeks 5, 6, 7 and 8 respectively).

A barrier to complete data collection throughout the project was that, in some cases, the time that the 
Average time for blood samples to be reported in Emergency Calls (minutes)



Graph 2 Run chart demonstrating median time for samples to be reported throughout project

samples were collected was not specified or clearly incorrect. For example, in a small minority of cases, the specified time that the blood sample was collected from the patient was later than the time the bloods were received in the lab. For these cases, parts of the data had to be excluded from analysis to minimise bias in results.

There was also some potential for bias and inaccuracy in the data collection. The sample reporting time specified on the electronic system only gave the time that the last result from the sample was reported. Clinicians are able to 'add-on' tests to bloods taken in the past 24 hours, and it is possible that in some cases these add-on tests skewed the data to suggest a very long reporting time. This potential for bias was addressed by using median times to analyse the data. Add-on tests are not routinely performed, and therefore it is unlikely that any such tests performed greatly skewed the median reporting times.

Another key caveat to data collection was that, given the information available, the time that results were reported on the electronic system was used as a surrogate marker for the clinical teams being aware of the results. This fails to take into account the fact that clinical staff are often busy with patients, and therefore may not regularly check to see if the results are available. As such, these timings are likely to underestimate the actual time taken for clinical teams to know the results. There was no way of overcoming this barrier to data collection as no timing record exists to say when a clinical team member checks the result. This problem was addressed by encouraging laboratory staff to relay the results from all Emergency calls to the clinical teams, as part of the quality improvement intervention. As such, the data collected following implementation is much more likely to accurately reflect the time that clinical teams became aware of results.

\section{LESSONS AND LIMITATIONS}

The greatest challenges faced during this project were staff engagement and data collection. When initially presenting the project idea and gathering the preimplementation data, we faced some hostility and scepticism from some members of staff and departments. In part, this was because the project was led by a junior doctor with limited experience working in the hospital. Perhaps more importantly, the project was perceived as a criticism of current working practices, particularly within the laboratory. These problems were eventually overcome with extensive engagement work, including numerous formal meetings and presentations, as well as informal discussions with staff to explain that the project was simply trying to make an improvement. Engaging with senior staff much earlier in the process, and recruiting them to the project, would have made the initial implementation a much simpler process.

Robust data collection also provided a challenge during the quality improvement implementation. Members involved in the project could only sporadically attend calls and directly collect data due to their other 
Graph 3 Overall median times

nime between samples received in lab and reported of each step of blood test reporting in emergency calls



work commitments. For this reason, we utilised existing databases of MET and Cardiac arrest calls compiled by resuscitation officers and critical care nurses. In a small number of cases, there was insufficient data in the database to allow for inclusion in the final analysis.

In addition, the project was limited to a short time period and as such there were not a large number of emergency calls from which to draw firm conclusions regarding the success of the project. It is possible that the observed improvement could have been due to random error or chance. This seems unlikely though given the drastic improvement in laboratory turnaround time for emergency samples. Further data collection over a longer period of time will help clarify this.

Our project was also limited in that each PDSA cycle involved multiple interventions. Therefore, although there was an overall improvement, it is not possible to ascertain the contribution of each intervention. Ideally, we would have tested each intervention individually through PDSA cycles.

Despite all clinical team members being aware of the project and buying into its goals, in addition to the educational interventions designed to encourage use of the forms, teams forgetting to use the forms remained an issue throughout the project. Over the course of the implementation phase, the forms were used in 19 out of 50 cases where blood samples were taken. This is understandable as emergency calls are often highly pressurised environments where team members are working quickly.
This issue was compounded by the fact that the forms were not conveniently located among the blood collecting equipment: a decision that was taken at the start of the project to minimise the risk of the forms being used inappropriately. Over more time, it is possible that this issue will improve as the use of the forms becomes more embedded in the emergency team culture. As demonstrated in Supplementary graph 2B, usage of the forms is associated with a greater improvement in reporting times.

\section{CONCLUSION}

This project arose from anecdotal concerns that delayed reporting of blood samples for critically ill patients was slowing the process of diagnosis and management, and therefore having a serious impact on patient care. By assessing the processes involved in blood sample reporting, a number of interventions were introduced that improved the time taken for samples to be reported for patients who had Cardiac Arrest or Medical Emergency calls put out for them.

The integrated nature of the interventions makes it difficult to ascertain which interventions were most important in generating the improvement seen. However, it is likely that the fast-tracking of samples flagged as coming from an emergency was very important, particularly given the heavy workload of the RSCH laboratory. This is supported by a study of laboratory 
turnaround times for emergency department samples, which identified sample delivery to the lab and order processing as the two major drivers for delay. ${ }^{8}$

There is no robust research exploring how outcomes are improved by more rapid diagnosis and reporting of laboratory tests. However, given the scientifically rational basis on which cardiac arrest and medical emergency teams are founded, it stands to reason that enabling clinicians to make quicker and better management decisions, by making results available faster, is positive for patient care. In this regard, the project met its original stated aim to speed up the reporting of blood tests and was not victim to 'project-creep', although it did not achieve the $50 \%$ improvement we had aimed for.

Another key issue will be whether the improvements seen with the project will be sustained. The simplistic nature of the interventions and the willingness of stakeholders to undertake them, coupled with the positive results demonstrated, will hopefully provide a solid foundation for sustained improvement.

Another positive outcome from the project is that it has utilised innovations already in place at the hospital to make an improvement. The morning medical emergency team meeting is a recent development that has aimed to improve communication and leadership among team members. It is run by the Critical Care team, who were instrumental in engaging other clinical staff members with the project, and as such provided an excellent forum in which the project could be presented to clinical staff and any issues addressed. We hope that others will be encouraged to take advantage of the supportive people, and systems, in place around them to undertake other quality improvement projects.

Acknowledgements Claire Cox, Gary Weaving, Denise Hedge, Sarah Goodhew, Owen Boyd, Jan Beddoe

\section{Declaration of interests Nothing to declare}

Ethical approval As per ethical guidelines set out nationally by the NHS Research Authority, and locally by Brighton and Sussex University Hospitals Trust, this project was exempt from ethical approval as it constituted an operational improvement activity and did not study human subjects.

Open Access This is an open-access article distributed under the terms of the Creative Commons Attribution Non-commercial License, which permits use, distribution, and reproduction in any medium, provided the original work is properly cited, the use is non commercial and is otherwise in compliance with the license. See:

- $h t t p: / / c r e a t i v e c o m m o n s . o r g / l i c e n s e s / b y-n c / 2.0 /$

- http://creativecommons.org/licenses/by-nc/2.0/legalcode

\section{REFERENCES}

1. Buist MD, Jarmolowski E, Burton PR, Bernard SA, Waxman BP, Anderson J. Recognising clinical instability in hospital patients before cardiac arrest or unplanned admission to intensive care. A pilot study in a tertiary-care hospital. Med J Aust. 1999;171:22-5.

2. Bellomo R, Goldsmith D, Uchino $S$ et al. Prospective controlled trial of effect of medical emergency team on postoperative morbidity and mortality rates. Critical care medicine. 2004;32:916-21.

3. Kerridge RK, Saul WP. The medical emergency team, evidence-based medicine and ethics. Med J Aust. 2003;179:313-5.

4. Kumar A, Roberts D, Wood KE et al. Duration of hypotension before initiation of effective antimicrobial therapy is the critical determinant of survival in human septic shock. Critical care medicine. 2006;34:1589-96.

5. Li L, Georgiou A, Vecellio E et al. The effect of laboratory testing on emergency department length of stay: a multihospital longitudinal study applying a cross-classified random-effect modeling approach. Academic emergency medicine : official journal of the Society for Academic Emergency Medicine. 2015;22:38-46.

6. Lee-Lewandrowski E, Corboy D, Lewandrowski K, Sinclair J, McDermot S, Benzer TI. Implementation of a Point-of-Care Satellite Laboratory in the Emergency Department of an Academic Medical Center. Archives of Pathology \& Laboratory Medicine. 2003;127:456-60.

7. Rooney KD, Schilling UM. Point-of-care testing in the overcrowded emergency department - can it make a difference? Critical Care. 2014;18:692.

8. Jalili M, Shalileh K, Mojtahed A, Mojtahed M, Moradi-Lakeh M. Identifying causes of laboratory turnaround time delay in the emergency department. Archives of Iranian medicine. 2012;15:759-63. 